\title{
Dynamics of test bodies in scalar-tensor theory and equivalence principle
}

\author{
Yuri N. Obukhov \\ Theoretical Physics Laboratory, Nuclear Safety Institute, \\ Russian Academy of Sciences, B.Tulskaya 52, 115191 Moscow, Russia \\ E-mail: obukhov@ibrae.ac.ru \\ Dirk Puetzfeld \\ ZARM, University of Bremen, \\ Am Fallturm, 28359 Bremen, Germany \\ E-mail: dirk.puetzfeld@zarm.uni-bremen.de,URL:http://puetzfeld.org
}

September 30, 2021

\begin{abstract}
How do test bodies move in scalar-tensor theories of gravitation? We provide an answer to this question on the basis of a unified multipolar scheme. In particular, we give the explicit equations of motion for pointlike, as well as spinning test bodies, thus extending the well-known general relativistic results of Mathisson, Papapetrou, and Dixon to scalar-tensor theories of gravity. We demonstrate the validity of the equivalence principle for test bodies.
\end{abstract}

Keywords: Scalar-tensor theories; Equations of motion; Equivalence principle.

\section{Introduction}

Scalar-tensor theories are considered to be close and viable generalizations of Einstein's general relativity theory. Since their introduction in [17, 18, 26] they attracted a lot of attention after the works of Brans and Dicke [5, 2, 3, 7, 8, with the scalar field interpreted as a variable gravitational coupling - for an overview of the history and results of scalar-tensor theories see [11, 4, 12, 24].

However, little attention was paid to the study of motion of extended test bodies in such theories. Following [3, 1, 27, the issue was thoroughly analyzed [6] in the framework of the post-Newtonian formalism. Here we present the test body dynamics for a very large class of scalar-tensor theories. 


\section{Scalar-tensor theory}

We study a class of scalar-tensor theories (along the lines of [6] ) with the action $I=\int d^{4} x \stackrel{J}{\mathfrak{L}}$ on the spacetime manifold with the metric $\stackrel{J}{g}_{i j}$. The Lagrangian density $\stackrel{J}{\mathfrak{L}}=\sqrt{-g}^{J} L$ has the following form

$$
\stackrel{J}{L}=\frac{1}{2 \kappa}\left(-F^{2} R(\stackrel{J}{g})+\stackrel{J}{g}^{i j} \gamma_{A B}^{J} \partial_{i} \varphi^{A} \partial_{j} \varphi^{B}-2 U^{J}\right)+L_{\mathrm{m}}\left(\psi, \partial \psi,{ }^{J}{ }_{i j}\right) .
$$

This extends the Brans-Dicke theory 28, 29] to the case with a multiplet of scalar fields $\varphi^{A}$ (capital indices $A, B, C=1, \ldots, N$ label the components of the multiplet). Here $\kappa=8 \pi G / c^{4}$ is Einstein's gravitational constant and

$$
F=F\left(\varphi^{A}\right), \quad \stackrel{J}{U}=\stackrel{J}{U}\left(\varphi^{A}\right), \quad \stackrel{J}{\gamma}_{A B}=\stackrel{J}{\gamma}_{A B}\left(\varphi^{A}\right) .
$$

The Lagrangian $L_{\mathrm{m}}\left(\psi, \partial \psi, \stackrel{J}{g}_{i j}\right)$ depends on the matter fields $\psi$.

The metric $\stackrel{J}{g}_{i j}$ determines angles and intervals in the Jordan reference frame. The Riemannian curvature scalar $R(\stackrel{J}{g})$ is constructed from the Jordan metric. With the help of the conformal transformation

$$
\stackrel{J}{g}_{i j} \longrightarrow g_{i j}=F^{2} \stackrel{J}{g}_{i j}
$$

we obtain the metric in the Einstein reference frame.

In the Einstein reference frame the Lagrangian density in the scalar-tensor theory reads $\mathfrak{L}=\sqrt{-g} L$ with

$$
L=\frac{1}{2 \kappa}\left(-R+g^{i j} \gamma_{A B} \partial_{i} \varphi^{A} \partial_{j} \varphi^{B}-2 U\right)+\frac{1}{F^{4}} L_{\mathrm{mat}}\left(\psi, \partial \psi, F^{-2} g_{i j}\right) .
$$

The scalar curvature $R(g)$ is constructed from the Einstein metric $g_{i j}$, and

$$
\gamma_{A B}=\frac{1}{F^{2}}\left(\gamma_{A B}^{J}+6 F_{, A} F_{, B}\right), \quad U=\frac{1}{F^{4}} \stackrel{J}{U} .
$$

The metrical energy-momentum tensor of matter is defined by $\sqrt{-g} t_{i j}:=$ $2 \partial\left(\sqrt{-g} L_{\text {mat }}\right) / \partial g^{i j}$. The Noether theorem yields the conservation law

$$
\nabla_{j} t^{k j}=\frac{1}{F}\left(4 t^{k j}-g^{k j} g_{m n} t^{m n}\right) \partial_{j} F=-V_{i j}{ }^{k} t^{i j} .
$$

For details see [20]. Here $V_{i j}{ }^{k}=A_{j} \delta_{i}^{k}-\frac{1}{4} g_{i j} A^{k}$, and $A_{i}:=\partial_{i} \log F^{-4}$.

\section{Equations of motion}

We derive the equations of motion in the Mathisson-Papapetrou-Dixon 19 21, 9, 10] approach by integrating the conservation law (6) using the geodesic 
expansion technique of Synge [25. With the world function $\sigma$ and the parallel propagator by $g^{y} x$, we introduce integrated moments to an arbitrary order $n=$ $0,1,2, \ldots$ by:

$$
\begin{aligned}
p^{y_{1} \ldots y_{n} y_{0}} & :=(-1)^{n} \int_{\Sigma(s)} \sigma^{y_{1}} \cdots \sigma^{y_{n}} g^{y_{0}} x_{0} \sqrt{-g} t^{x_{0} x_{1}} d \Sigma_{x_{1}}, \\
k^{y_{2} \ldots y_{n+1} y_{0} y_{1}} & :=(-1)^{n} \int_{\Sigma(s)} \sigma^{y_{1}} \cdots \sigma^{y_{n}} g^{y_{0}}{ }_{x_{0}} g^{y_{1}} x_{1} \sqrt{-g} t^{x_{0} x_{1}} w^{x_{2}} d \Sigma_{x_{2}} .
\end{aligned}
$$

We use a condensed notation so that $y_{n}$ denotes indices at the point $y$ which we associate with the world-line $y(s)$ of a test body, parametrized by the proper time $s$. The integrals are performed over spatial hypersurfaces $\Sigma(s)$.

\section{Pole-dipole equations of motion}

In the pole-dipole approximation, an extended body is characterized by the multipole moments $p^{a}, p^{a b}, k^{a b}, k^{a b c}$. Using the general multipolar scheme [23] we derive the equations of motion for these moments:

$$
\begin{aligned}
0 & =k^{(a|c| b)}-v^{(a} p^{b) c} \\
\frac{D}{d s} p^{a b} & =k^{b a}-v^{a} p^{b}-V_{d c}{ }^{b} k^{a c d}, \\
\frac{D}{d s} p^{a} & =-V_{c b}{ }^{a} k^{b c}-V_{d c}{ }^{a} ; k^{b c d}-\frac{1}{2} R^{a}{ }_{c d b}\left(k^{b c d}+v^{d} p^{b c}\right) .
\end{aligned}
$$

Here $v^{a}:=d y^{a} / d s$ denotes the normalized four-velocity of a body. Since $k^{a[b c]}=$ 0 , we can solve (9) to find explicitly

$$
k^{a b c}=v^{a} p^{c b}+v^{c} p^{[a b]}+v^{b} p^{[a c]}+v^{a} p^{[b c]} .
$$

Plugging this into (10) and (11), we obtain the generalized Mathisson-PapapetrouDixon system

$$
\begin{aligned}
\frac{D \mathcal{P}^{a}}{d s} & =\frac{1}{2} R_{b c d}^{a} v^{b} \mathcal{J}^{c d}-\xi \nabla^{a} F^{-4}-\xi^{b} \nabla_{b} \nabla^{a} F^{-4}, \\
\frac{D \mathcal{J}^{a b}}{d s} & =-2 v^{[a} \mathcal{P}^{b]}-2 \xi^{[a} \nabla^{b]} F^{-4} .
\end{aligned}
$$

Here, following [22, 23, 20, we introduce the generalized total energy-momentum 4 -vector and the generalized total angular momentum by

$$
\begin{aligned}
\mathcal{P}^{a} & :=F^{-4} p^{a}+p^{b a} \nabla_{b} F^{-4}, \\
\mathcal{J}^{a b} & :=F^{-4} L^{a b} .
\end{aligned}
$$

The orbital angular moment is defined by $L^{a b}:=2 p^{[a b]}$, and we denoted

$$
\xi^{a}=-\frac{1}{4} g_{b c} k^{a b c}, \quad \xi=-\frac{1}{4} g_{a b} k^{a b} .
$$




\section{Monopolar equations of motion}

At the monopolar order, the only nontrivial moments are $p^{a}$, and $k^{a b}$. The system (9)-(11) then reduces to

$$
\begin{aligned}
0 & =k^{b a}-v^{a} p^{b}, \\
\frac{D p^{a}}{d s} & =-V_{c b}{ }^{a} k^{b c} .
\end{aligned}
$$

Making use of $k^{[a b]}=0$, the first equation yields $v^{[a} p^{b]}=0$, hence we have

$$
p^{a}=M v^{a}
$$

with the mass $M:=v^{a} p_{a}$. Substituting (18) and (20) into (19) we find $\xi=$ $-\frac{v^{a} p_{a}}{4}=-\frac{M}{4}$ and

$$
\frac{D v^{a}}{d s}=-\left(g^{a b}-v^{a} v^{b}\right) \frac{\nabla_{b} F}{F} .
$$

Quite remarkably, the dynamics of an extended test body in the monopole approximation is independent of body's mass. For a trivial coupling function $F$, equation (21) reproduces the general relativistic result. Interestingly, the mass of a body is not constant: $M=F^{3} M_{0}$ with $M_{0}=$ const.

\section{Conclusions}

Our main result is the system (13)-(14) that describes the dynamics of extended test bodies in scalar-tensor gravity. In the monopolar case, our analysis revealed a surprisingly simple equation of motion (21). In contrast to geodesic motion in General Relativity, freely falling massive test bodies in scalar-tensor gravity experience an additional force, determined by the new scalar degrees of freedom encoded in the function $F$.

A remarkable feature of (21) is the prediction that all massive test bodies move in the same way, independently of their mass. We thus demonstrate the validity of the equivalence principle in scalar-tensor gravity. This is consistent with the previous observation [15, 16] that the total scalar charge of a body is equal to its mass when the scalar field self-interactions are neglected. The latter is in agreement with the test body assumption that underlies the Mathisson-Papapetrou-Dixon approach. When one goes beyond the test body approximation, however, the scalar charge is no longer equal to the mass and a further study is needed to fix their relation [13, 14].

Acknowledgements D.P. was supported by the Deutsche Forschungsgemeinschaft (DFG) through the grant SFB 1128 (geo-Q).

\section{References}

[1] P. Bergmann, Int. J. Theor. Phys., 1:25, 1968. 
[2] C. Brans, Phys. Rev., 125:2194, 1962.

[3] C. Brans, Phys. Rev., 125:388, 1962.

[4] C. Brans, arXiv:gr-qc/0506063, 2005.

[5] C. Brans and R. H. Dicke, Phys. Rev., 124:925, 1961.

[6] T. Damour and G. Esposito-Farèse, Class. Quantum Grav., 9:2093, 1992.

[7] R. H. Dicke, Phys. Rev., 125:2163, 1962.

[8] R. H. Dicke, The theoretical significance of experimental relativity (Gordon and Breach: New York, 1964).

[9] W. G. Dixon, Nuovo Cimento, 34:317, 1964.

[10] W. G. Dixon, Phil. Trans. R. Soc. Lon. A, 277:59, 1974.

[11] Y. Fujii and K. Maeda, The scalar-tensor theory of gravity (Cambridge University Press: Cambridge, 2003).

[12] H. Goenner, Gen. Rel. Grav., 44:2077, 2012.

[13] S. E. Gralla, Phys. Rev. D, 81:084060, 2010.

[14] S. E. Gralla, Phys. Rev. D, 87:104020, 2013.

[15] L. Hui and A. Nicolis, Phys. Rev. Lett., 105:231101, 2010.

[16] L. Hui, A. Nicolis, and C. W. Stubbs, Phys. Rev. D, 80:104002, 2009.

[17] P. Jordan, Schwerkraft und Weltall (Vieweg: Braunschweig, 1955).

[18] P. Jordan, Z. Phys., 157:112, 1959.

[19] M. Mathisson, Acta Phys. Pol., 6:163, 1937.

[20] Y. N. Obukhov and D. Puetzfeld, Phys. Rev. D, 90:104041, 2014.

[21] A. Papapetrou, Proc. Roy. Soc. Lond. A, 209:248, 1951.

[22] D. Puetzfeld and Y. N. Obukhov, Phys. Rev. D, 88:064025, 2013.

[23] D. Puetzfeld and Y. N. Obukhov, Phys. Rev. D, 90:084034, 2014.

[24] T. P. Sotiriou, Lect. Notes Phys., Ed. E. Papantonopoulos, 892:3, 2015.

[25] J. L. Synge, Relativity: The general theory (North-Holland: Amsterdam, 1960).

[26] Y. Thiry, J. Math. Pures et Appl., sér. 9, 30:275, 1951.

[27] R. V. Wagoner, Phys. Rev., 12:3209, 1970.

[28] C. M. Will, Theory and experiment in gravitational physics (Cambridge University Press: Cambridge, 1993).

[29] C. M. Will, Living Reviews in Relativity, 17(4), 2014. 